\title{
Capillary torque on a particle rotating at an interface
}

\author{
Abhinav Naga, Doris Vollmer, and Hans-Jürgen Butt \\ Max Planck Institute for Polymer Research, \\ Ackermannweg 10, 55128 Mainz, Germany
}

May 27, 2021

\section{Supporting Information}

\section{Calculation of $k$ factor for a cubic variation of $\cos \Theta(\alpha)$}

In this section, the $k$ factor in Eq. 6 is evaluated using a cubic function to describe $\cos \Theta(\alpha)$.

The contact angle function has to satisfy four boundary conditions in order to be both smooth and continuous:

- $\cos \Theta(\alpha=-\pi / 2)=\cos \Theta_{\mathrm{A}}$,

- $\cos \Theta(\alpha=\pi / 2)=\cos \Theta_{\mathrm{R}}$,

- $\frac{d(\cos \Theta)}{d \alpha}=0$ at $\alpha=-\pi / 2$, and

- $\frac{d(\cos \Theta)}{d \alpha}=0$ at $\alpha=\pi / 2$.

The last two conditions are required in order to have a smooth contact angle variation at the extremities. Since there are four boundary conditions, the function describing the contact 
angle variation must have four independent parameters. A cubic polynomial fulfills this requirement:

$$
\cos \Theta(\alpha)=a \alpha^{3}+b \alpha^{2}+c \alpha+d
$$

where $a, b, c$, and $d$ are independent variables. To find these variables in terms of the advancing and receding angles, we use the four boundary conditions listed above and obtain,

$$
\begin{aligned}
a & =\frac{2}{\pi^{3}}\left(\cos \Theta_{\mathrm{A}}-\cos \Theta_{\mathrm{R}}\right) \\
b & =0 \\
c & =-\frac{3}{2 \pi}\left(\cos \Theta_{\mathrm{A}}-\cos \Theta_{\mathrm{R}}\right) \\
d & =\frac{1}{2}\left(\cos \Theta_{\mathrm{A}}+\cos \Theta_{\mathrm{R}}\right) .
\end{aligned}
$$

Therefore, the equation describing $\Theta(\alpha)$ is

$$
\cos \Theta(\alpha)=\frac{2}{\pi^{3}}\left(\cos \Theta_{\mathrm{A}}-\cos \Theta_{\mathrm{R}}\right) \alpha^{3}-\frac{3}{2 \pi}\left(\cos \Theta_{\mathrm{A}}-\cos \Theta_{\mathrm{R}}\right) \alpha+\frac{1}{2}\left(\cos \Theta_{\mathrm{A}}+\cos \Theta_{\mathrm{R}}\right) .
$$

This equation is valid for $-\pi / 2<\alpha<\pi / 2$.

The expression for the magnitude of the capillary torque is (negative of Eq. 19 from the Appendix in the main manuscript):

$$
\begin{aligned}
M & =\gamma R^{2} \sin \phi \int_{0}^{2 \pi} \cos \Theta(\alpha) \sin \alpha d \alpha \\
& =\gamma R \frac{L}{2} \int_{0}^{2 \pi} \cos \Theta(\alpha) \sin \alpha d \alpha,
\end{aligned}
$$

where $R$ is the radius of the particle and $L=2 R \sin \phi$ is the diameter of the contact line. 
To find the $k$ factor corresponding to a cubic variation in $\cos \Theta(\alpha)$, we first rewrite the integral in Eq. S8 such that the limits of integration match the range for which our definition of $\cos \Theta(\alpha)$ is valid $(-\pi / 2$ to $\pi / 2)$,

$$
\begin{aligned}
\frac{M}{\gamma R L} & =\frac{1}{2} \int_{0}^{2 \pi} \cos \Theta(\alpha) \sin \alpha d \alpha \\
& =\frac{1}{2} \int_{-\pi}^{\pi} \cos \Theta(\alpha) \sin \alpha d \alpha \\
& =\int_{-\frac{\pi}{2}}^{\frac{\pi}{2}} \cos \Theta(\alpha) \sin \alpha d \alpha
\end{aligned}
$$

The last step is due to symmetry of $\Theta(\alpha)$ about the $y z$ plane.

Next, we substitute Eq. S1 into Eq. S9 to obtain

$$
\begin{aligned}
\frac{M}{\gamma R L} & =\int_{-\frac{\pi}{2}}^{\frac{\pi}{2}} \sin \alpha\left[a \alpha^{3}+c \alpha+d\right] d \alpha \\
& =\left[\left(\frac{3 \pi^{2}}{2}-12\right) a+2 c+0\right] \\
& =\frac{24}{\pi^{3}}\left(\cos \Theta_{\mathrm{R}}-\cos \Theta_{\mathrm{A}}\right) .
\end{aligned}
$$

Therefore, $k=24 / \pi^{3}$. The following results were used to perform the integration:

$$
\begin{aligned}
\int_{-\frac{\pi}{2}}^{\frac{\pi}{2}} \alpha \sin \alpha d \alpha & =\int_{-\frac{\pi}{2}}^{\frac{\pi}{2}} \alpha \frac{d(-\cos \alpha)}{d \alpha} d \alpha \\
& =[-\alpha \cos \alpha]_{-\frac{\pi}{2}}^{\frac{\pi}{2}}--\int_{-\frac{\pi}{2}}^{\frac{\pi}{2}} \cos \alpha d \alpha \\
& =2,
\end{aligned}
$$

and 


$$
\begin{aligned}
\int_{-\frac{\pi}{2}}^{\frac{\pi}{2}} \alpha^{3} \sin \alpha d \alpha & =\int_{-\frac{\pi}{2}}^{\frac{\pi}{2}} \alpha^{3} \frac{d(-\cos \alpha)}{d \alpha} d \alpha \\
& =\left[-\alpha^{3} \cos \alpha\right]_{-\frac{\pi}{2}}^{\frac{\pi}{2}}--3 \int_{-\frac{\pi}{2}}^{\frac{\pi}{2}} \alpha^{2} \cos \alpha d \alpha \\
& =3 \int_{-\frac{\pi}{2}}^{\frac{\pi}{2}} \alpha^{2} \frac{d(\sin \alpha)}{d \alpha} d \alpha \\
& =3\left[\alpha^{2} \sin \alpha\right]_{-\frac{\pi}{2}}^{\frac{\pi}{2}}-3 \int_{-\frac{\pi}{2}}^{\frac{\pi}{2}} 2 \alpha \sin \alpha d \alpha \\
& =\frac{3 \pi^{2}}{2}-12 .
\end{aligned}
$$

